\title{
Key Parts of Transmission Line Detection Using Improved YOLO v3
}

\author{
Tu Renwei ${ }^{1}$, Zhu Zhongjie ${ }^{1}$, Bai Yongqiang ${ }^{1}$, Gao Ming ${ }^{2}$, and Ge Zhifeng ${ }^{2}$ \\ ${ }^{1}$ College of Information and Intelligence Engineering, Zhejiang Wanli University, China \\ ${ }^{2}$ Ninghai Power Supply Company Limited, State Grid Corporation of Zhejiang, China
}

\begin{abstract}
Unmanned Aerial Vehicle (UAV) inspection has become one of main methods for current transmission line inspection, but there are still some shortcomings such as slow detection speed, low efficiency, and inability for low light environment. To address these issues, this paper proposes a deep learning detection model based on You Only Look Once (YOLO) v3. On the one hand, the neural network structure is simplified, that is the three feature maps of YOLO v3 are pruned into two to meet specific detection requirements. Meanwhile, the K-means++ clustering method is used to calculate the anchor value of the data set to improve the detection accuracy. On the other hand, 1000 sets of power tower and insulator data sets are collected, which are inverted and scaled to expand the data set, and are fully optimized by adding different illumination and viewing angles. The experimental results show that this model using improved YOLO v3 can effectively improve the detection accuracy by $6.0 \%$, flops by $8.4 \%$, and the detection speed by about $6.0 \%$.
\end{abstract}

Keywords: Deep learning, YOLO v3, electric tower, insulator.

Received October 31, 2019; accepted February 4, 2021

https://doi.org/10.34028/iajit/18/6/1

\section{Introduction}

With the rapid development of the economy, the demand for industrial and domestic electricity has increased substantially. Safe and stable operation of transmission lines is of great significance to the regional social and economic development. In order to eliminate safety hazards before power failure, daily inspections of transmission lines are essential [8]. China has a vast territory, complex terrain, varied meteorological conditions, and most of the power lines pass through the mountains, making the inspections greatly difficult. Especially after natural disasters such as earthquakes and typhoons, the inspection environment has become more complicated. For manual inspection, there are some shortcomings, such as major safety hazards, long inspection time and low efficiency. Meanwhile, inspectors are labor-intensive and expensive. The Unmanned Aerial Vehicle (UAV) power inspection is a detection method based on machine learning and target detection algorithm, which enables inspection without direct contact with the power tower. Compared with manual inspection, UVA inspection has the advantages of low risk, high efficiency and low cost $[2,10]$. Therefore, the UAV inspection technology has received extensive attention in the power industry.

In recent years, artificial intelligence has been rapidly developed, especially machine vision and deep learning technology, making it feasible for UAV to inspect power systems and evaluate high-voltage line conditions [1]. The target detection algorithm using the deep convolutional neural network to extract the target features can be used for the detection of key components of the transmission line [16]. At present, there are two types of mainstream deep learning target detection algorithms, one is a region-based target detection algorithm, and the other is a regressionbased target detection algorithm. The region-based target detection algorithms include Faster Regions with CNN features (R-CNN) [4, 17] and Mask RCNN [18]. The detection accuracy of such algorithms is high, but the detection speed is still slow, which cannot be adapted to the high-intensity real-time inspection requirements. Comparatively, the target detection algorithms based on regression include You Only Look Once (YOLO) [5] and Single Shot MultiBox Detector (SSD) [9] have fast detection speed and can meet real-time detection requirements, but the detection accuracy of small targets needs to be improved. In particular, it is easy for the UAV to detect the insulator over long distances, which is prone to missed detection and false detection. At present, there is no public data set of key parts of the power tower, so the challenge of related detection comes from both the algorithm itself and the limited data set.

Aiming at the particularity of location identification of power towers and insulators, this paper proposes a deep learning detection model based on YOLO v3, which optimizes the neural network architecture of YOLO v3 and creates a new data set. The specific contributions are as follows:

1. The structure of the neural network is simplified. 
2. Relatively large and medium-sized target data sets are established, and two feature extraction networks with different sizes of receptive fields are designed to better extract the features of large and mediumsized targets. The feature maps used to detect large and medium-sized targets in the original YOLO v3 are retained. A feature map used to improve the detection performance of small targets is clipped. In this way, the total amount of calculation is reduced and the detection speed is improved.

3 . For the newly created data set, the K-means++ clustering method is used to recalculate the anchor value of the training set to improve the detection accuracy of the detection target. A number of 1000 power tower and insulator data sets were created by UAV shooting and online collection of pictures, including 500 power towers and insulator pictures, and the data set images are reversed and scaled [19]. The influence of the background of the data set, illumination and other factors on the training of the model is fully considered, and the optimization of the data set is realized.

To validate the performance of the proposed method, we compare results with YOLO v3. The results show that improved YOLO v3's average IoU increased by $5.32 \%$, precision increased by $6 \%$, total computation decreased by $8.4 \%$, and detection speed increased by $6 \%$, which basically realized the requirement of realtime high-precision detection.

\section{YOLO v3}

YOLO was proposed by Redmon and Farhadi [12] of the University of Washington in 2015. After YOLO v1, YOLO v2 to the latest development of YOLO v3 [6], making the latest models have advantages in detection speed and small target detection that other deep learning models do not have. It has a fast detection speed and high detection accuracy. In terms of basic image feature extraction, YOLO v3 uses a network structure called Darknet-53, which has 53 convolutional layers. In order to extract features better, residual block are used [3], and shortcut links are set up between some layers. Inside the entire structure of YOLO v3, there is no pooling layer and full connectivity layer. Therefore, when the convolutional layer is transmitted, the scalar size transformation is realized by changing the convolution kernel moving step size, such as stride $=(2,2)$, which is equivalent to dividing the image side length by 2 , so that the area is the original 1/4. During the entire convolution process, YOLO v3 has undergone 5 down samplings, after the third down sampling, it outputs $52 \times 52 \times(416 / 8=52)$ feature maps, and after the 4th down sampling, outputs $26 \times 26 \times(416 / 16=26)$ feature maps. Similarly, a feature map of $13 \times 13 \times(416 / 32=13)$ is output after the fifth down sampling [14].
As shown in Figure 1, Darknet-53 is composed of five residual blocks, each of which consists of a plurality of residual units, and a residual unit is constructed by inputting residual operations with two Darknet-convolution, Batch normalization and Leaky relu activation function (DBL) units. Among them, the DBL unit contains convolution, batch normalization and leaky relu activation function, as shown in Figure 2. By introducing a residual unit, the depth of the network can be deeper to avoid the gradient disappearing [20].

YOLO v3 down sampled the input image 5 times and predicted the target in the last 3 down samplings. The last three down samplings contain three feature maps for scale target detection. The small feature map provides deep semantic information, while the large feature map provides location information of the target. The small feature map is merged with the large feature map to detect both small targets and large targets. Overall, YOLO v3 maps input images to 3 scale output tensors. A picture with the size of $416 \times 416$, each grid of each scale has 3 a priori boxes, so there are $13 \times 13 \times 3+26 \times 26 \times 3+52 \times 52 \times 3=10647$ prediction boxes in total [15].

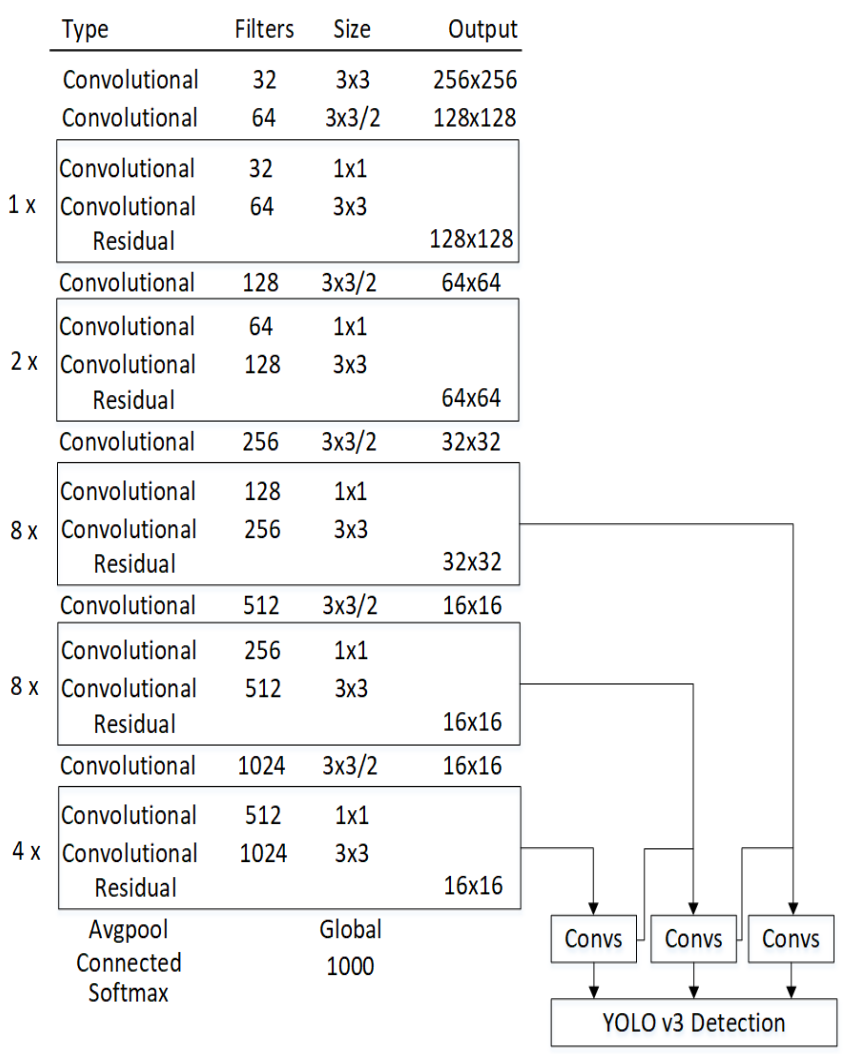

Figure 1. The overall structure of YOLO v3.
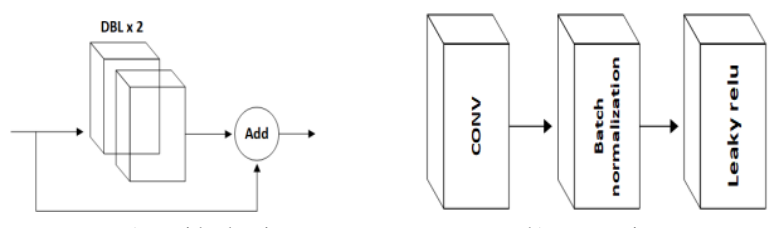

a) Residual unit.

b) DBL unit.

Figure 2. Darknet-53 structural unit. 


\section{Overall Design}

Based on YOLO v3, a real-time detection method for key components of transmission lines is proposed, as shown in Figure 3. Firstly, a new data set of key components of the transmission line is established. Images of different brightness are added to optimize the data set, and then the data set is labeled with YOLOMark. In order to get more accurate anchors, Kmeans++ is used to cluster the labeled data set. Finally, the model is trained and one model is saved every 1000 iterations. The one with the best performance is selected as the final detection model. At this time, realtime detection can be achieved by inputting the UAV inspection video into the detection model.

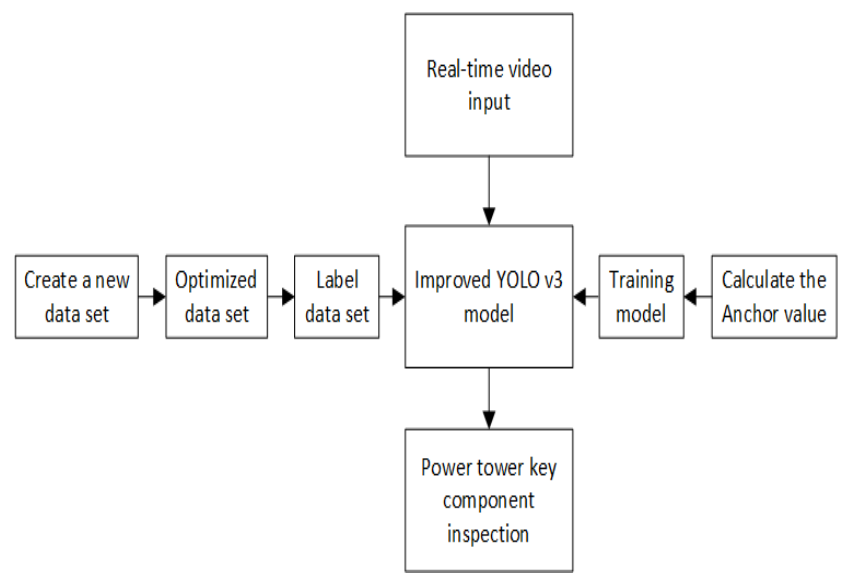

Figure 3. Overall design.

\subsection{Establishment and Optimization of Data Sets}

Training samples for neural networks usually require at least a few thousand. If the sample data is too small, it will affect the accuracy and reliability of the detection. Since there is no publicly available detection database for the key parts of the transmission tower, this paper has established a 1000-volume data set by collecting pictures of the online pictures and UAV. There are 500 pictures of the power tower and the insulator, and the picture contains a variety of complicated situations: multiple power towers and multiple insulators appear in a single picture, or one power tower has multiple insulators.

These images contain different illumination, different detection target shooting angles, different resolutions, different detection backgrounds, etc., which meet the requirements of sample diversity, so that they are purposefully optimized. The optimization of the sample is important for the improvement of the robustness of the algorithm. If the brightness factor of the data set is not considered, the training of the model using only a single-illuminated image will lead to unreliable detection performance in a dark environment. Also, complex background and different resolution images are added to the data set, so that the detection model can be better trained. Part of the data set shown in Figure 4:

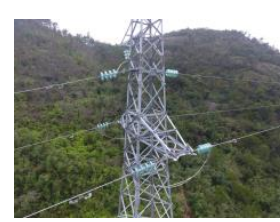

a) Front view.

c) Bottom view.

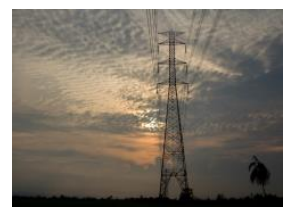

d) Low light.

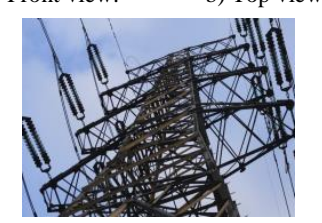

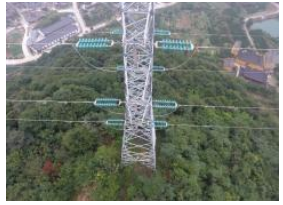

b) Top view.

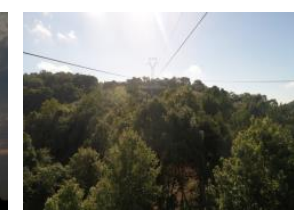

e) Strong light.

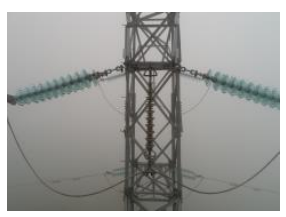

f) Foggy.

Figure 4. Part of the data set.

At the same time, it is necessary to standardize the training set annotation technique. The low-resolution image and small target have great influence on the model training. Its influence is reflected in the oscillation and detection accuracy of the Loss curve. The deep learning network is prone to errors in the extraction of low-resolution images and small target features, making it more prone to miss detection and false detection. Therefore, in this paper, the annotation of the small target of the low-resolution image and the minimum target of the high-resolution image is discarded at the time of labelling, so as to improve the robustness of the model.

\subsection{Pruning of Neural Network Structure}

YOLO v3 has a very good characteristic for the detection of small targets. In this paper, the actual detection of power towers and insulators has low demand for small target detection. The feature map of $52 \times 52$ in YOLO v3 is to extract the shallow neural network features to achieve the detection of small targets, so the pruning of the layer does not theoretically affect the detection results.

Figure 5 shows the structures before and after pruning where (a) is the original structure of YOLO v3 and (b) is the structure after pruning. The two share the same backbone network Darknet-53 which consists of the res1, res2, res4, res8 residual blocks, six DBLs and a convolutional layer where the res8 is used twice aiming to make the network deeper to 
improve the performance of feature extraction and to construct multiple feature maps of different scales to improve the detection performance of targets with different sizes as well. Since the power towers and insulators studied in this paper belong to relatively large and medium sizes, the targets in the established data set are all large and medium sizes, with very few small sizes. However, the output of the three receptive fields of different sizes of YOLO v3 is aimed at the detection of large, medium and small size targets. For the purpose of this paper, it is more reasonable to retain the output for detecting large and medium-sized targets. For the detection of key parts of the transmission tower, deleting some parts that have little effect on the detection will help reduce the calculation amount and improve the detection speed.

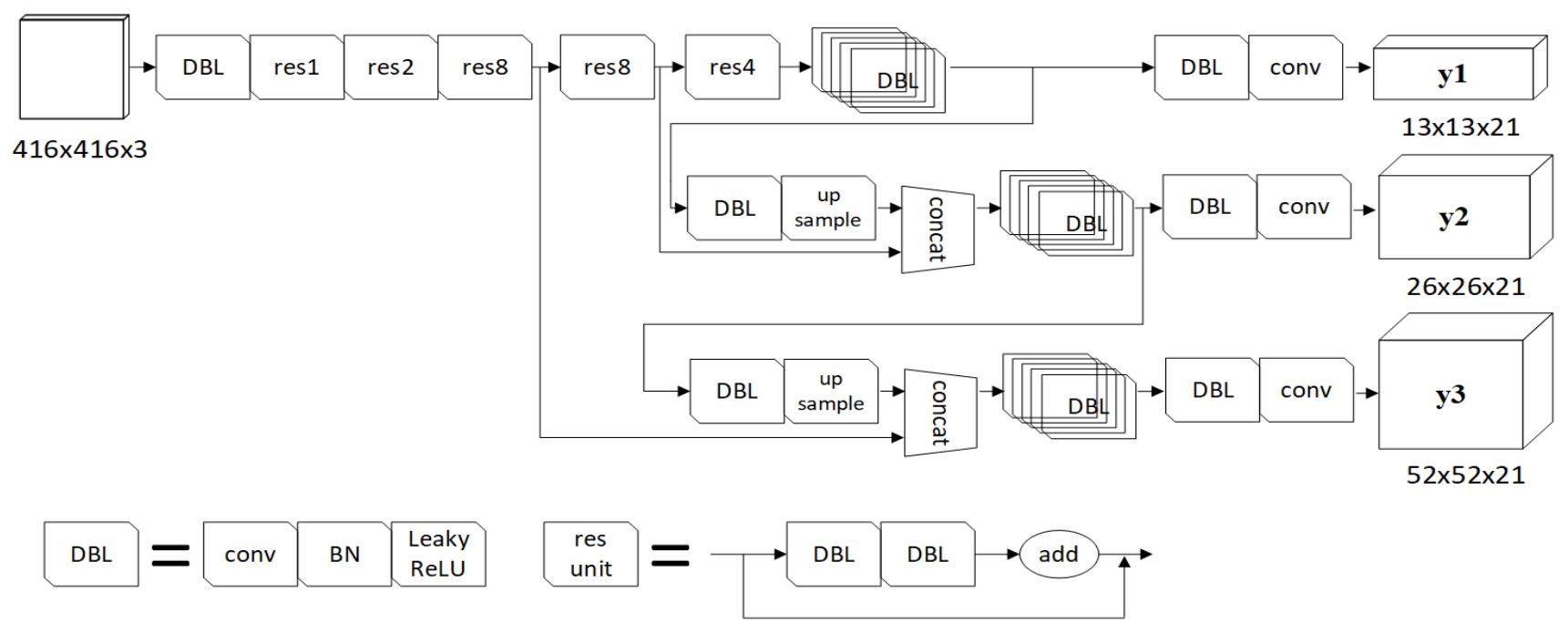

a) YOLO v3.
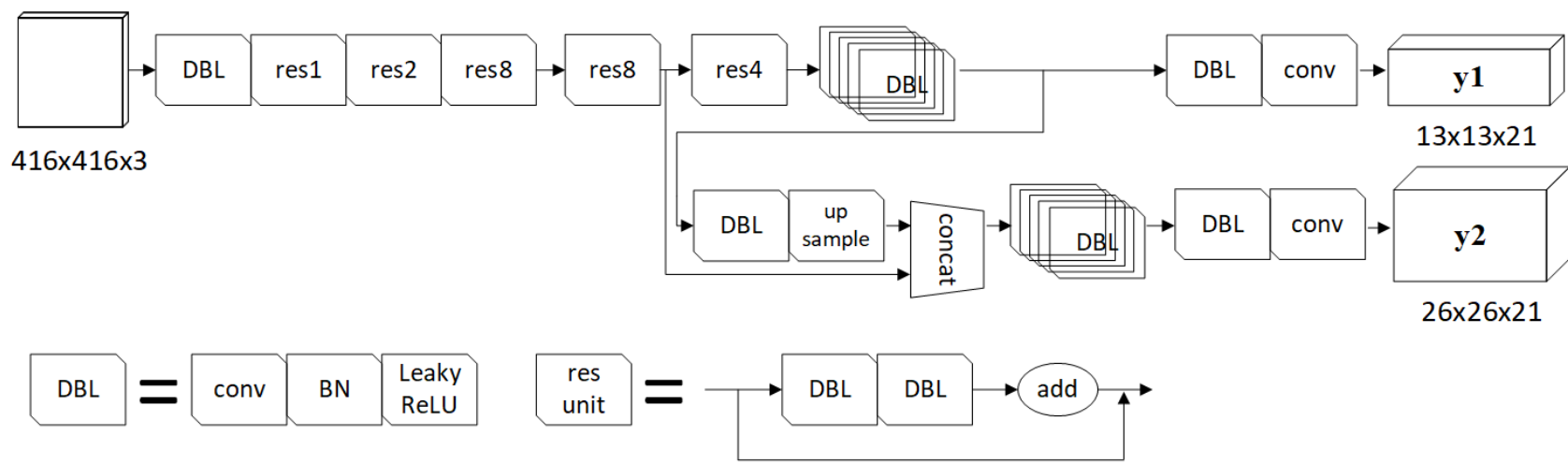

b) Improved YOLO v3 after pruning.

Figure 5. Comparison before and after pruning.

\subsection{Selection of Accurate Anchor}

Anchors are used to predict the bounding box. In the Fast R-CNN, $128 \times 128,256 \times 256,512 \times 512$ are used to transform 1:1, 1:2, 2:1 in three scales, and a total of 9 anchors are used to predict the box. Each anchor predicts about 2,000 frames, making the detection rate much higher. YOLO v2 began to increase the anchor mechanism [7], 9 anchors are added in YOLO v3. Anchors of YOLO v3 are 10, 13, 16, 30, 33, 23, 30, 61, $62,45,59,119,116,90,156,198,373,326$, which are the original values form VOC data sets. There are 20 types of targets in the Visual Object Classes (VOC). Each target has a different size and a large gap. If the original values of anchor are used for the newly established data set, it is obviously unreasonable. Therefore, the new data set is clustered to get anchors to improve detection accuracy.
The K-means++ algorithm is a typical distancebased clustering algorithm. The degree of similarity is evaluated according to the distance, that is, the closer the distance between two objects is, the greater the similarity is. Clusters are made up of objects that are close together, so a compact and independent cluster is the ultimate goal. K-means++ $[11,13]$ is used to calculate anchors, because the use of Euclidean distance will make the larger bounding boxes produce more errors than the smaller bounding boxes. After getting more accurate anchors through clustering, good IoU scores can be obtained.

$$
I o U=\frac{\text { DetectionResult } \cap \text { GroundTruth }}{\text { DetectionResult } \cup \text { GroundTruth }}
$$

$I o U$ is the ratio of the intersection and the union of the prediction box and the label box. The larger the ratio, the better the detection performance of the 
detection model and the higher the accuracy, as shown in Figure 6. In this paper, we set 1 to 9 cluster centers for the data set, and cluster the resulting anchor and IoU values, as shown in Table 1:

Table 1. K-means++ clustering results analysis.

\begin{tabular}{|c|c|c|}
\hline $\mathbf{K}$ & Anchor & Avg IoU \\
\hline 1 & 54,118 & $21.21 \%$ \\
\hline 2 & $30,61,138,324$ & $38.31 \%$ \\
\hline 3 & $26,47,63,182,173,364$ & $43.83 \%$ \\
\hline 4 & $24,42,57,129,95,357,232,353$ & $47.19 \%$ \\
\hline 5 & $29,24,13,56,84,63,34,167,153,356$ & $58.53 \%$ \\
\hline 6 & $12,48,33,27,30,145,92,67,91,344,226,359$ & $59.58 \%$ \\
\hline 7 & $12,50,28,22,59,50,30,151,132,89,88,348,217,368$ & $60.15 \%$ \\
\hline 8 & $11,45,29,23,24,109,63,50,43,215,136,91,101,367,234,364$ & $61.06 \%$ \\
\hline 9 & $11,44,27,22,22,90,58,44,34,182,116,84,69,328,133,374,267,349$ & $61.88 \%$ \\
\hline
\end{tabular}

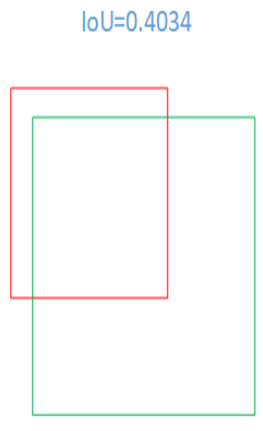

Poor
$\mathrm{loU}=0.7330$

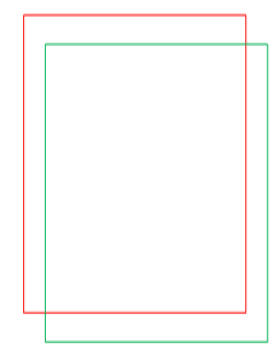

Good
IoU $=0.9264$

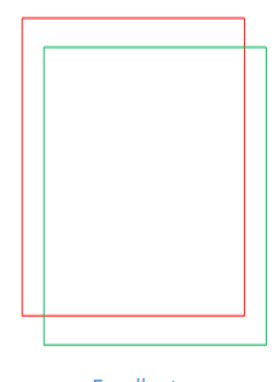

Excellent
Figure 6. IoU schematic.

The above table shows the values of different anchors and different Avg IoUs obtained by setting different numbers of cluster centers. With the increase of the cluster center point, Avg IoU shows an increasing trend, and the increasing speed is fast and slow, and gradually converges. The minimum value of Avg IoU is $21.21 \%$ and the maximum is $61.88 \%$. From the above table, it can be seen from the above table that when $K=6$, it is the inflection point position of the change of the entire Avg IoU, and then it starts to converge. In order to obtain the best detection speed and detection result, the anchor value of $\mathrm{K}=6$ is selected in this paper: $(12,48),(33,27),(30,145),(92,67),(91$, $344),(226,359)$.

\section{Experimental Results and Analysis}

Due to the large amount of sample data required to train the deep learning network, high-performance GPU parallel computing is needed to improve the training and testing speed. The experimental development environment selected is as follows:

1. Hardware environment:

CPU: intel i7 8700, 3.2GHz

GPU: NVIDIA GeForce GTX1060 6G

RAM: $16 \mathrm{G}$

2. Software environment

Operating system: Win 10

Deep Learning Network Framework: Darknet53
Software: Visual Studio 2015, OpenCV 3.2.0, CUDA 9.1, cuDNN 7.1

In order to better control the model and obtain better detection results, the training parameters of YOLO v3 are optimized for the detection target characteristics and the data volume limitation of this paper, as shown in Table 2. A total of two types of power tower and insulator detection targets are set in the paper. The number of iterations for each type of detection target is 4000, a total of 8000 iterations of training, and the training set is reversed and scaled to expand the data volume. The input resolution is $416 \times 416$ and multiscale training is turned on. Table 2. Training parameter settings.

Table 2. Training parameter settings.

\begin{tabular}{|l|c|c|c|c|c|c|}
\hline Algorithm & Batch & Subdivisions & Input & Decay & $\begin{array}{c}\text { Learning } \\
\text { rete }\end{array}$ & $\begin{array}{c}\text { Max } \\
\text { batches }\end{array}$ \\
\hline $\begin{array}{l}\text { Improved } \\
\text { YOLO v3 }\end{array}$ & 64 & 16 & $416 \times 416$ & 0.0005 & 0.001 & 8000 \\
\hline
\end{tabular}

The learning rate determines the speed at which the weights are updated. If the setting is too large, the result will exceed the optimal value. Too small will make the falling speed too slow. So set a dynamically changing learning rate to get a better model. When the number of iterations is from 0 to 6,400 , the learning rate is set to 0.001 . When the number of iterations is between 6400 and 7200, the learning rate is set to 0.0001 . When the number of iterations is 7200 to 8000 , the learning rate is set to 0.00001 . Throughout the training process, the learning rate attenuation reaches 100 times.

The training got the Loss curve in the above figure. The previous training set learning rate $=0.001$ makes Loss fall rapidly. From the 2400th iteration, the Loss slowdown becomes slower and more stable, and it converges from the 6400th iteration. Finally, the loss value converges at 0.7676 , and an ideal small sample training result is obtained. Figure 7 shows the comparison of the Loss curves before and after the data set optimization. Figure 7-a) shows the Loss before data set optimization and Figure 7-b) shows the Loss after data set optimization. The abscissa is the number of iterations and the ordinate is the Loss value. In Figure 7-b), there is a very large oscillation when the model is iterated to 800 times, and the curve price reduction is accompanied by large fluctuations when iterating 800 to 1600 times. When iterating 6400 times, it appears larger oscillation again. Explain the impact of data sets on model training: As the learning rate decays, instability occurs. The Loss in Figure 7-b) shows a more uniform and stable attenuation change throughout the iteration, without violent oscillations, and eventually Loss tends to converge. 


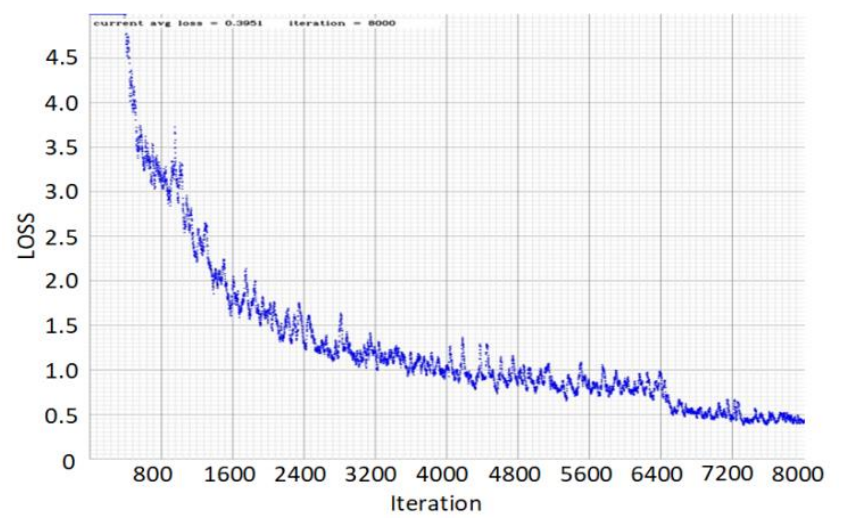

a) Loss before data set optimization.

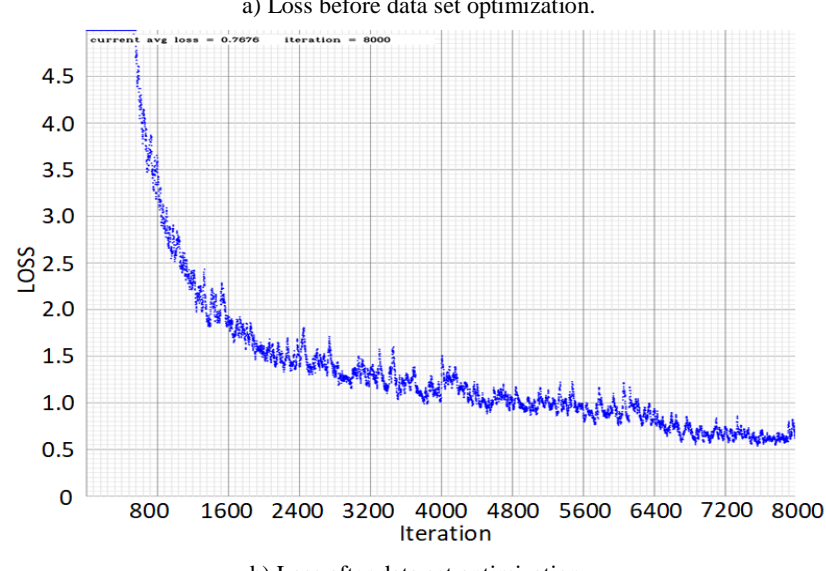

b) Loss after data set optimization.

Figure 7. Comparison of Loss curves before and after data set optimization.

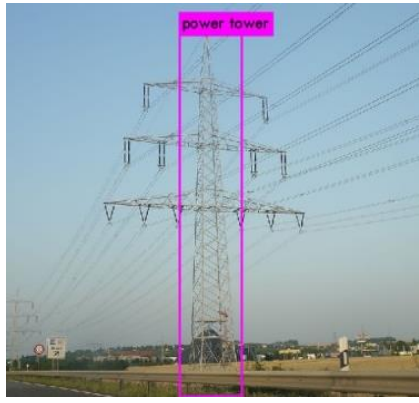

a) Before optimization.

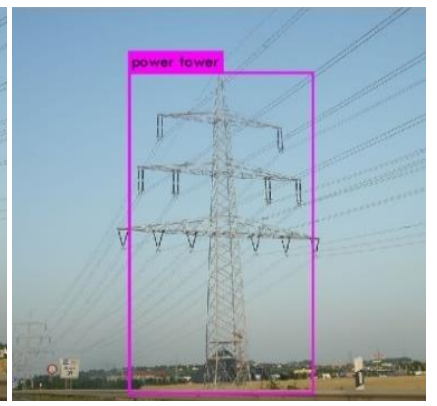

b) After optimization
Figure 8. Comparison of test results before and after optimization of anchor value.

Figure 8-a) shows the test result of improved YOLO v3 using the original anchors, and Figure 8-b) shows the test result of improved YOLO v3 using the anchors clustered by K-means++ in the newly created data set. It is not difficult to find that the positioning of the detection frame on the left is not accurate. The power tower is not completely framed on the left and right sides of the detection frame, and the target under the detection frame does not fit the target, which is not compact enough. The detection frame on the right is basically able to frame the target well, and the detection frame is positioned very accurately.

The reliability of the model has been verified by a large number of image tests. The experimental results are as follows: The improved YOLO v3 has good performance for the detection of power towers and insulators, meeting the requirements of real-time detection. Compared with the existing algorithms, the proposed algorithm reduces the amount of calculation and improves the detection speed while ensuring the accuracy of detection. The data set has been optimized so that it still has high detection performance under low light conditions, especially in the scenes before and after extreme weather or natural disasters.
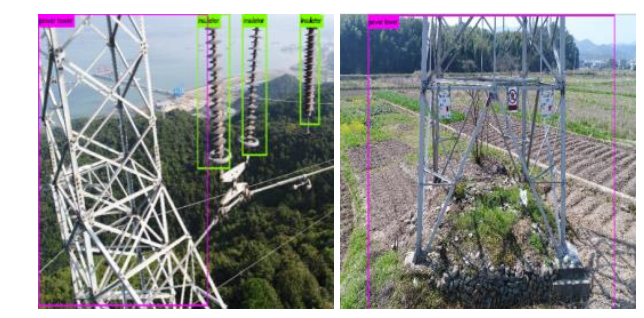

a) One power tower and insulators. b) The base of power tower.

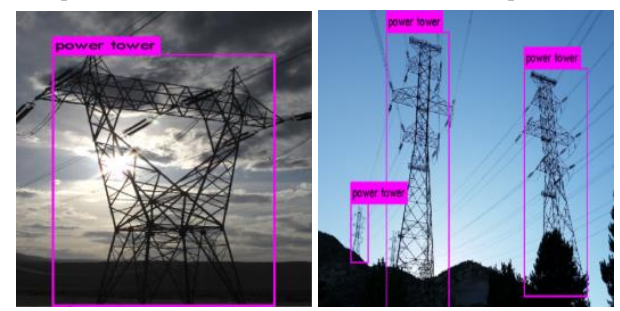

c) One power tower in the dark
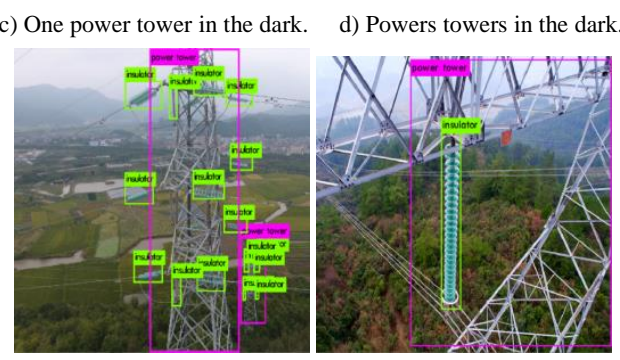

e) Powers towers and insulators.

f) Part of power tower.

Figure 9. Partial test results.

In this paper, six representative experimental results are selected for analysis. The above figure shows the detection ability of the proposed algorithm for single target and multi-target scenes. In Figure 9-a), one power tower and three insulators are detected at the same time. The positioning of the detection frame is very accurate, and there is no missed detection or false detection. In Figure 9-b), it is the detection result of the base of power tower, checking whether the power tower collapses or falls. The test results show that the model has certain generalization ability. Figure 9-c) and Figure 9-d) are the results of power tower detection in dark environment. Figure 9-e) shows the detection results of multiple power towers and multiple insulators. The experimental results show the reliability of the proposed algorithm for single large target under low light and multi-target under low light.

Tables 3 and 4 show the comparison between YOLO v3 and improved YOLO v3. The improved YOLO v3 recall is 0.45 higher than YOLO v3 by 0.03 , F1-score is basically the same, the Avg IoU increased by $5.32 \%$, the precision increased by $6.0 \%$. At the same time, Total BFLOPs dropped by about $8.4 \%$, making detection speed up $6.0 \%$. 
Table 3. Comparison of test results.

\begin{tabular}{|c|c|c|c|c|c|}
\hline Algorithm & Recall & F1-score & Avg IoU & Prescision & Speed \\
\hline YOLO v3 & 0.42 & 0.56 & $62.06 \%$ & $82 \%$ & $33.2 \mathrm{~ms} /$ frame \\
\hline $\begin{array}{l}\text { Improved } \\
\text { YOLO v3 }\end{array}$ & 0.45 & 0.57 & $67.38 \%$ & $88 \%$ & $31.2 \mathrm{~ms} /$ frame \\
\hline
\end{tabular}

Table 4. Comparison of detection speed and calculation amount.

\begin{tabular}{|c|c|c|}
\hline Algorithm & Speed & Total BFLOPs \\
\hline YOLO v3 & $33.2 \mathrm{~ms} /$ frame & 65.297 \\
\hline Improved YOLO v3 & $31.2 \mathrm{~ms} /$ frame & 59.819 \\
\hline Improved rate & $6.0 \%$ & $-8.4 \%$ \\
\hline
\end{tabular}

\section{Conclusions}

Based on YOLO v3, an improved algorithm for power tower and insulator detection is proposed in this paper. A data set containing relatively large and medium-sized targets is established, and two-way convolutional networks with different sizes of receptive fields are designed to better extract features. Also, the use of Kmeans++ makes the calculation of anchors more accurate. The model detection accuracy is $88 \%$ and the detection speed is $31.2 \mathrm{~ms} /$ frame, which show the reliability of the proposed algorithm for the detection of power towers and insulators. In the future, we will explore more advanced algorithms for real-time identification of damage to key parts of transmission lines. For example, real-time identification of the collapse of transmission towers and breakage of insulators can prevent transmission line accidents from happening earlier.

\section{Acknowledgement}

This work was supported in part by the National Natural Science Foundations of China (No. 61671412); Zhejiang Provincial Natural Science Foundation of China (No. LY19F010002, LY21F010014); Fundamental and Commonweal Projects of Zhejiang Province (No. LGN20F010001); General Scientific Research Project of Zhejiang Education Department (No. Y201941122); Ningbo Municipal Projects for Leading and Top Talents (No. NBLJ201801006); Natural Science Foundation of Ningbo, China (No. 2018A610053, 202003N4323); Innovation and consulting project from Ninghai Power Supply Company, State Grid Corporation of Zhejiang, China; Fundamental Research Funds for Zhejiang Provincial Colleges and Universities; School-level Research and Innovation Team of Zhejiang Wanli University.

\section{References}

[1] Bhola R., Krishna N., Ramesh K., Senthilnath J., and Anand G., "Detection of the Power Lines in UAV Remote Sensed Images Using SpectralSpatial Methods," Journal of Environmental Management, vol. 206, pp. 1233-1242, 2018.

[2] Chen B. and Miao X., "Distribution Line Pole Detection and Counting Based on YOLO Using
UAV Inspection Line Video," Journal of Electrical Engineering and Technology, vol. 15, no. 1, pp. 441-448, 2020.

[3] Chen H., He Z., Shi B., and Zhong T., "Research on Recognition Method of Electrical Components Based on YOLO V3," IEEE Access, vol. 7, pp. 157818-157829, 2019.

[4] Fang F., Li L., Zhu H., and Lim J., "Combining Faster R-CNN and Model-Driven Clustering for Elongated Object Detection," IEEE Transactions on Image Processing, vol. 29, no. 1, pp. 20522065, 2020.

[5] Hsu W. and Lin W., "Ratio-and-Scale-Aware YOLO for Pedestrian Detection," IEEE Transactions on Image Processing, vol. 30, pp. 934-947, 2021.

[6] Ju M., Luo H., Wang Z., Hui B., and Chang Z., "The Application of Improved YOLO V3 in Multi-Scale Target Detection," Applied Sciences, vol. 9, pp. 1-14, 2019.

[7] Kim K., Kim P., Chung Y., and Choi D., "MultiScale Detector for Accurate Vehicle Detection in Traffic Surveillance Data," IEEE Access, vol. 7, pp. 78311-78319, 2019.

[8] Liu Z., Wang X., and Liu Y., "Application of Unmanned Aerial Vehicle Hangar in Transmission Tower Inspection Considering the Risk Probabilities of Steel Towers," IEEE Access, vol. 7, pp. 159048-159057, 2019.

[9] Lu X., Ji J., Xing Z., and Miao Q., "Attention and Feature Fusion SSD for Remote Sensing Object Detection," IEEE Transactions on Instrumentation and Measurement, vol. 70, pp. $1-9,2021$.

[10] Miao X., Liu X., Chen J., Zhuang S., Fan J., and Jiang H., "Insulator Detection in Aerial Images for Transmission Line Inspection Using Single Shot Multibox Detector," IEEE Access, vol. 7, pp. 9945-9956, 2019.

[11] Maghawry A., Omar Y., and Badr A., "SelfOrganizing Map vs Initial Centroid Selection Optimization to Enhance K-Means with Genetic Algorithm to Cluster Transcribed Broadcast News Documents," The International Arab Journal of Information Technology, vol. 17, no. 3, pp. 316-324, 2020.

[12] Redmon J. and Farhadi A., "YOLOv3: An Incremental Improvement," arXiv preprint arXiv:1804.02767, 2018.

[13] Ren S. and Zhang X., "Synthesizing Conjunctive and Disjunctive Linear Invariants by K-means++ and SVM," The International Arab Journal of Information Technology, vol. 17, no. 6, pp. 847856, 2020.

[14] Tian Y., Yang G., Wang Z., Wang H., Li E., and Liang Z., "Apple Detection During Different Growth Stages in Orchards using the Improved 
YOLO-V3 Model," Computers and Electronics in Agriculture, vol. 157, pp. 417-426, 2019.

[15] Tian Y., Yang G., Wang Z., Li E., and Liang Z., "Detection of Apple Lesions in Orchards Based on Deep Learning Methods of CycleGAN and YOLOV3-Dense," Journal of Sensors, vol. 2019, pp. 13, 2019.

[16] Wang S., Jiang F., Zhang B., Ma R., and Hao Q., "Development of UAV-Based Target Tracking and Recognition Systems," IEEE Transactions on Intelligent Transportation Systems, vol. 21, no. 8, pp. 3409-3422, 2020.

[17] Wang K. and Liu M., "Object Recognition at Night Scene Based on DCGAN and Faster RCNN," IEEE Access, vol. 8, pp. 193168-193182, 2020.

[18] Xi D., Qin Y., Luo J., Pu H., and Wang Z., "Multipath Fusion Mask R-CNN with Double Attention and its Application into Gear Pitting Detection," IEEE Transactions on Instrumentation and Measurement, vol. 70, pp. 111, 2021.

[19] Zhang L., Liu J., Zhang B., Zhang D., and Zhu C., "Deep Cascade Model-Based Face Recognition: When Deep-Layered Learning Meets Small Data," IEEE Transactions on Image Processing, vol. 29, pp. 1016-1029, 2020.

[20] Zhou L., Wei S., Cui Z., Fang J., Yang X., and Ding W., "Lira-YOLO: A Lightweight Model for Ship Detection in Radar Images," Journal of Systems Engineering and Electronics, vol. 31, no. 5, pp. 950-956, 2020.

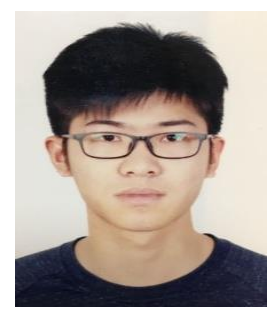

Tu Renwei received his B.S. degrees from Jiaxing University, China, in 2017, and now he is a master's student at Zhejiang Wanli University. His research interests mainly include digital video processing and application.

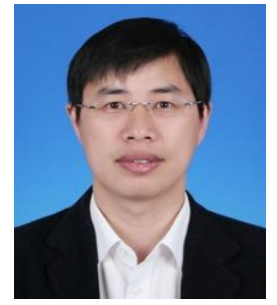

Zhu Zhongjie received a Ph.D. degree in electronics science and technology from Zhejiang University, China, in 2004. He is currently a professor with the Faculty of Electronics and Information Engineering, Zhejiang Wanli University, China. His research interests mainly include video compression and communication, image analysis and understanding, watermarking and information hiding, and 3D image signal processing.

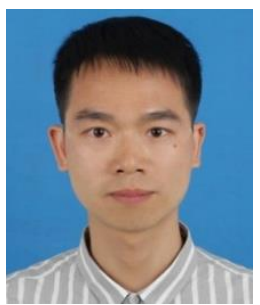

Bai Yongqiang received his B.S. and M.S. degrees from Zhengzhou University, China, in 2006 and 2009 respectively, and received his Ph.D. degree from Ningbo University, China, in 2019. He is now a researcher in Zhejiang Wanli University, China. His research interests mainly include data hiding, digital watermarking and image processing.

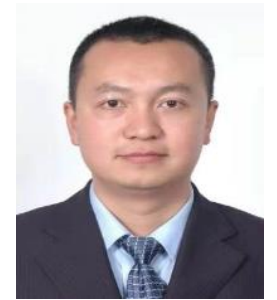

Gao Ming received M.S. degrees from Zhejiang University, China, in 2012. He is currently the executive director of Ninghai Power Supply Company Limited, State Grid Corporation of Zhejiang, China. His research interests mainly include power automation, artificial intelligence and its applications.

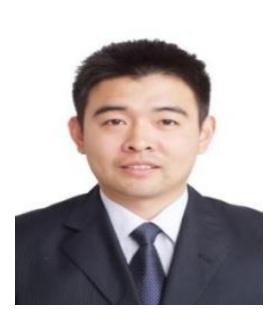

Ge Zhifeng received B.S. degrees from Changsha University of Science and Technology, China, in 2005. He is currently the director of the development and construction department of Ninghai Power Supply Company Limited, State Grid Corporation of Zhejiang, China. His research interests mainly include power automation, artificial intelligence and its applications. 